\title{
Transplantation of epidermal cell sheets by endoscopic balloon dilatation to avoid esophageal re-strictures: initial experience in a porcine model
}

Authors

Institutions
Shinichiro Kobayashi ${ }^{1,2}$, Nobuo Kanai $^{2}$, Nobuyuki Tanaka ${ }^{2,3}$, Masanori Maeda ${ }^{2}$, Takahiro Hosoi ${ }^{2,4}$, Fumio Fukai ${ }^{4}$, Susumu Eguchi', Masayuki Yamato ${ }^{2}$

Institutions are listed at the end of article. submitted 23. April 2016 accepted after revision 1. August 2016

\section{Bibliography}

DOI http://dx.doi.org/

10.1055/s-0042-116145

Published online: 28.10.2016

Endoscopy International Open 2016; 04: E1116-E1123

(c) Georg Thieme Verlag KG Stuttgart · New York

E-ISSN 2196-9736

\section{Corresponding author}

\section{Nobuo Kanai, MD PhD}

Institute of Advanced

Biomedical Engineering and Science

Tokyo Women's Medical

University

8-1 Kawada-cho

Shinjuku-ku

Tokyo 162-8666

Japan

Fax: +81-3-33596046

kanai.nobuo@twmu.ac.jp
Background and study aims: Epidermal cell sheet (ECS) transplantation immediately after aggressive endoscopic submucosal dissection (ESD) has been shown to be safe and effective in the prevention of esophageal strictures. This study evaluated the feasibility of ECS transplantation after endoscopic balloon dilation (EBD) in a porcine model. Methods: Six pigs underwent circumferential esophageal ESD under general anesthesia. Two weeks later, two pigs underwent EBD and transplantation of an autologous ECS, two underwent EBD alone, and two underwent endoscopic observation only (control).

Results: The two pigs in the transplantation group underwent six ECS transplants after EBD with five

\section{Introduction \\ $\nabla$}

Esophageal strictures are frequent complications after aggressive endoscopic submucosal dissection (ESD) for early stage esophageal cancer and adenocarcinoma [1,2]. However, most patients develop refractory esophageal strictures after extensive ESD. Although there are several treatments for refractory strictures, their utility is limited [3-6].

A cell-based tissue engineering approach involving the transplantation of autologous oral epithelial cell sheets or epidermal cell sheets (ECSs) to the sites of esophageal ESD has been employed to treat strictures in animal models $[7,8]$. Moreover, a clinical study has shown that transplantation of ECSs is safe and potentially effective [9]. Nevertheless, a refractory esophageal stricture has developed in a patient who underwent extensive ESD, defined as a $10 \mathrm{~cm}$, full-circumferential ESD [9]. Because re-strictures after endoscopic balloon dilation (EBD) may be due to tear re-adhesion after dilatation, we hypothesized that transplantation of ECSs to the tear after EBD would prevent tear re-adhesion and refractory esophageal strictures. However, difficulties were of the six (83\%) being successful, as shown by engraftment of transplanted ECSs after 7 days. No adverse events were observed. Stricture rates were lower in the two transplanted pigs (55\% and $60 \%$ ) than in the control (92.2\% and $87.7 \%$ ) and EBD-treated $(71.7 \%$ and $78.2 \%)$ pigs. Infiltration of inflammatory cells was significantly lower in the transplanted pigs than in the control and EBD-treated pigs.

Conclusion: Preliminary results indicate the stability of the ECS transplantation procedure and the engraftment of transplanted ECS in the tears after EBD. This proof-of-concept study suggests that covering tears with ECSs after EBD may avoid restrictures.

encountered while transplanting ECSs to the sites of post-EBD tears using conventional methods, suggesting the requirement for novel procedures. This study describes a novel method of ECS transplantation and demonstrates the engraftment of transplanted ECSs in the tears after EBD in a porcine model. A rapid prototyping technique, which included a three-dimensional computer-aided design (3D CAD) system and 3D printer, was used in the development of this method.

\section{Materials and methods \\ $\nabla$}

Experimental animals

Six miniature pigs (6 months old, $16-20 \mathrm{~kg}$ ) were purchased from the Nippon Institute for Biological Science (Tokyo, Japan). Experimental procedures were performed under general anesthesia. All animal experiments were approved by the Committee for Animal Research of Tokyo Women's Medical University (approval number: $11-512013)$. 


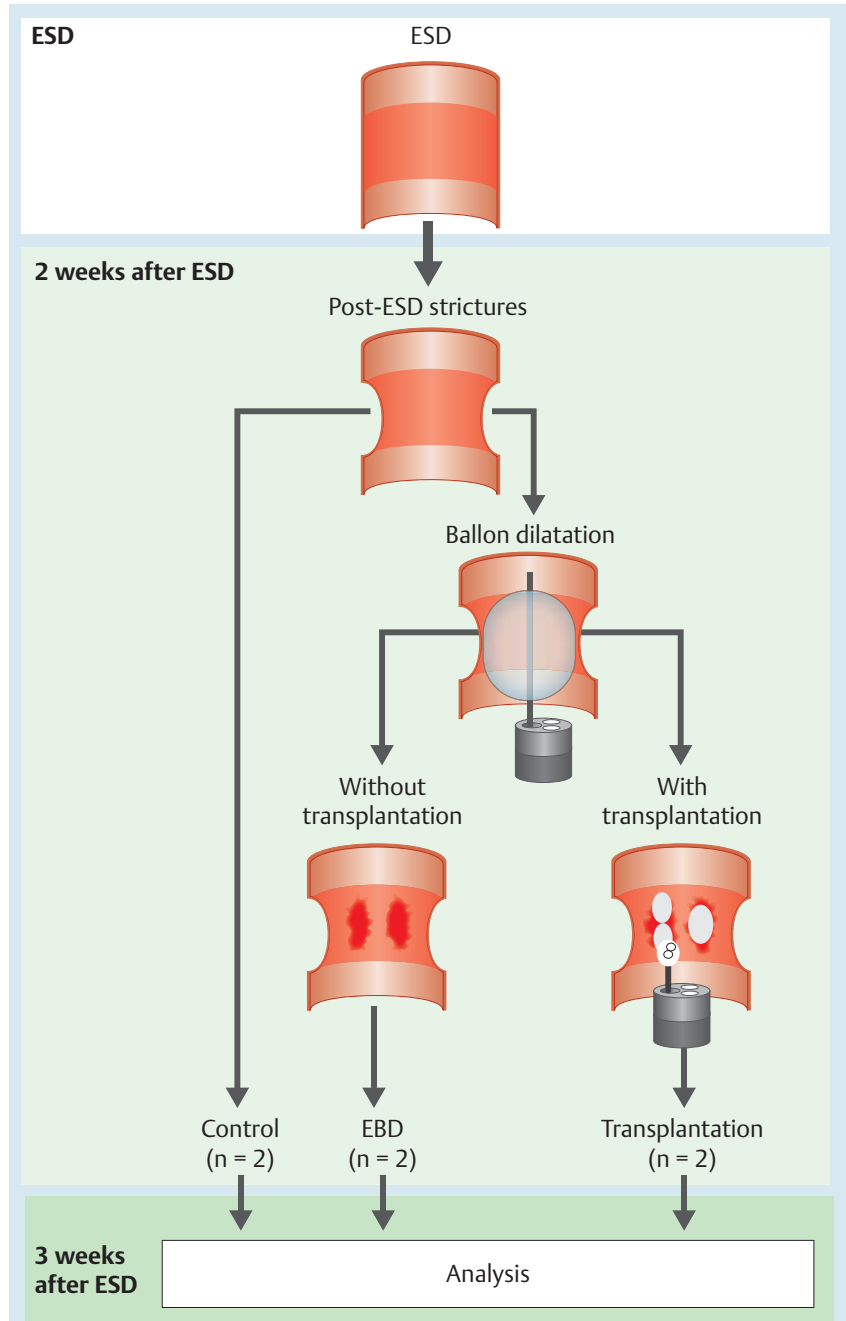

Fig. 1 Study design. Six pigs were prospectively divided into control, endoscopic balloon dilation (EBD), and transplantation groups. All pigs underwent endoscopic submucosal dissection (ESD). Two pigs were observed after ESD (Control group), two underwent EBD alone (EBD group), and two underwent EBD followed by transplantation of epidermal cell sheets (ECSs) (transplantation group). Three weeks after ESD, the six pigs were euthanized for further analyses.

\section{Study design}

All six pigs underwent esophageal ESD to trigger the development of esophageal strictures, and all six developed severe esophageal strictures at 2 weeks after ESD. The six pigs were prospectively divided into three groups, with two pigs in each. Control pigs underwent endoscopic observation without any treatment after ESD. Pigs in the EBD group underwent EBD, and pigs in the transplantation group underwent EBD followed by transplantation of ECSs. Three weeks after ESD, the six pigs were euthanized because the pigs in the control and EBD groups lost their appetites and were in a poor condition at $>3$ weeks after ESD ( $\bullet$ Fig. 1). The primary end point was to evaluate the safety and feasibility of ECS transplantation onto tears after EBD, whereas the secondary end points were engraftment of transplanted ECSs and prevention of re-stricture after EBD. Re-strictures after EBD were evaluated twice in each pig.

\section{Development of a novel transplantation device}

A part of the device was fabricated automatically by a $3 \mathrm{D}$ printer (Objet 350 ${ }^{\mathrm{TM}}$; Stratasys, Eden Prairie, Minnesota, United States) from biocompatible plastic material (MED610; Stratasys) $(\bullet$ Fig. 2 a). The device was introduced into a nylon tube through the forceps channel ( $\bullet$ Fig. $\mathbf{2 b}$ ).

\section{Preparation of ECSs}

Skin biopsies were obtained from the pigs. Keratinocytes were separated and seeded onto temperature-responsive cell culture inserts (CellSeed, Tokyo, Japan) in keratinocyte culture medium [10]. After cultivation for 14 days, keratinocyte cell sheets were harvested from cell culture inserts by reducing the temperature to $20^{\circ} \mathrm{C}(\nabla$ Fig. 2 c, d $)$.

\section{Endoscopic submucosal dissection}

The ESD procedure has been described previously [8]. Briefly, artificial ulcerations ( $360^{\circ}$ in range and $3 \mathrm{~cm}$ in length) were made in the lower esophagus using an endoscope (GIF-XQ260 or GIFHQ290; Olympus, Tokyo, Japan) and the hook-knife ESD method. A high-frequency electrosurgical generator (VIO300D ERBE; Elektromedizin, Tübingen, Germany) was set in the end cutting mode (effect 2).

\section{Endoscopic balloon dilatation}

Two weeks after esophageal ESD, pinhole strictures had developed in all pigs. Four slits were made in each pig using the hook knife or an insulation-tipped knife (IT-knife-2, Olympus), and the esophageal strictures were dilated using an endoscopic balloon device (HBD-W-10-11-12; Boston Scientific Co., Natick, Massachusetts, United States), allowing the endoscope to pass easily through the section of the esophagus treated with EBD.

\section{Endoscopic transplantation of ECSs}

After EBD, an EMR tube (Create Medic, Tokyo, Japan) was inserted into the esophagus of each animal. The ECS transplantation device performs three actions: suction, air ejection, and air flow $(\checkmark$ Video 1). The harvested ECS was gently placed on the device. For transport from the mouth to the ulcer site, suction was applied to the ECS with the syringe. When it reached the target site, the ECS was released by air ejection and spread by continuous air flow. The number of ECSs transplanted during each session was calculated to cover one-third of each EBD ulceration. The procedure times were measured from the endoscopic transport of the ECS to ECS transplantation at the ulcer sites.

\section{Postoperative care}

Beginning at 1 day after ESD, all pigs were provided with food and water. Pigs that underwent EBD or ECS transplantation were fasted for 2 days postoperatively and subsequently allowed free access to solid foods and water. Initially, solid foods were provided. Semisolid foods were provided when the solid diet could not pass into the stomach, and dysphagia and vomiting developed. Each animal was evaluated daily by animal care staff. Dysphagia was evaluated before ESD and at 2 and 3 weeks after ESD using a modified Mellow and Pinkas score [11]. Briefly, 0 was defined as the absence of dysphagia, 1 as the ability to eat some solid foods, 2 as the ability to eat semisolids only, 3 as the ability to swallow liquids only, and 4 as complete dysphagia. 


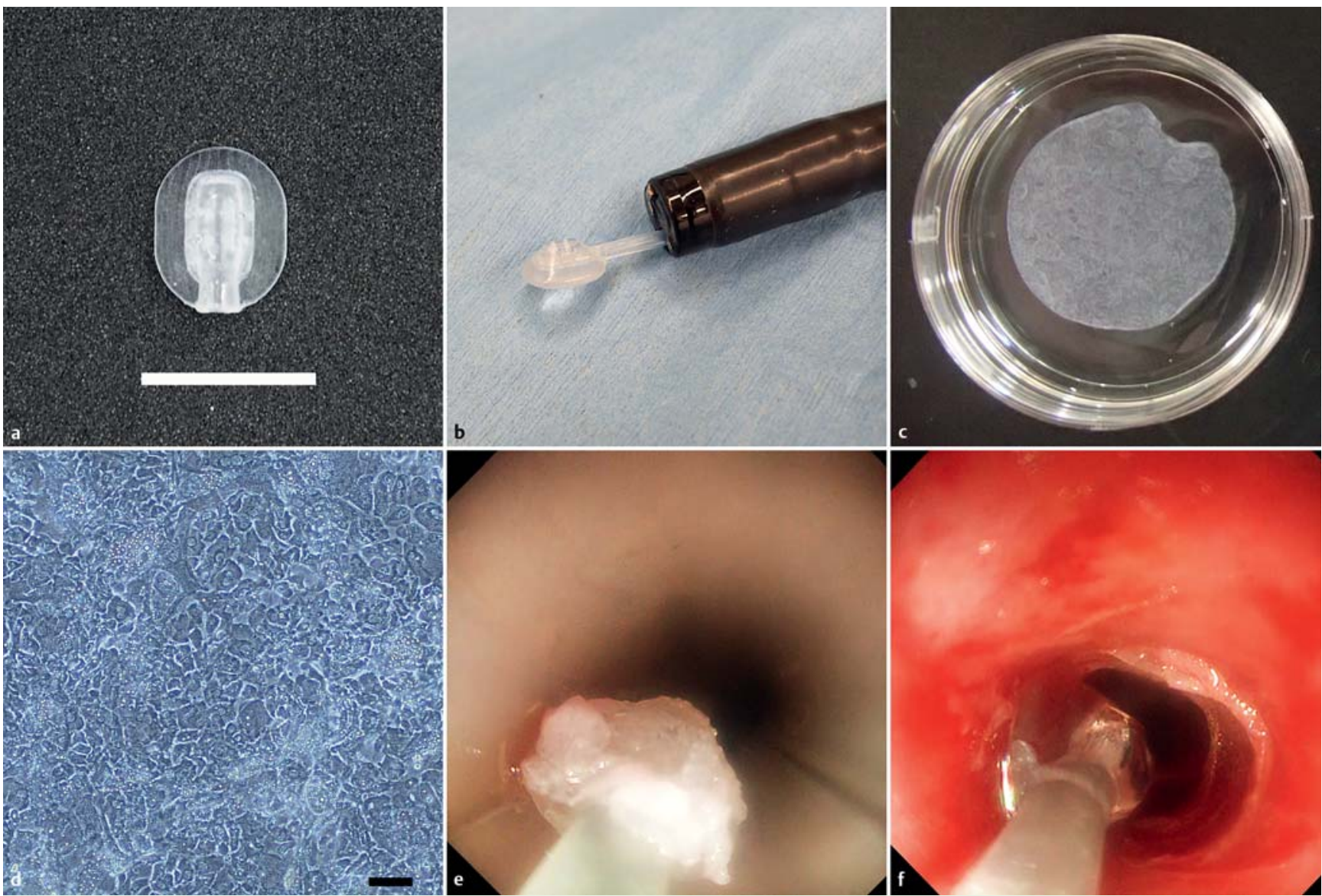

Fig. 2 Transplantation of epidermal cell sheets (ECSs) using the newly developed device. a The head of the device was fabricated automatically by a 3D printer. Scare bar indicates $10 \mathrm{~mm}$. b The head docked with the catheter in front of the endoscope. c A tissue-engineered ECS $\left(4.2 \mathrm{~cm}^{2}\right)$ formed by primary cells isolated from pig skin fragments $\left(2.5 \mathrm{~cm}^{2}\right)$ after 2 weeks of culture. $\mathbf{d}$ H\&E staining of epidermal cells in the ECSs, indicating a cobblestone-like pattern. Scale bar indicates $20 \mu \mathrm{m}$. (e, f) Actions of the developed device with two modes: transport and release. e The transport mode was set at suction with the ECS transported from the mouth onto the tears after endoscopic balloon dilation (EBD). $\mathbf{f}$ The release mode was set at air ejection. The release procedure enabled accurate placement of the ECS onto the artificial ulcer after EBD.

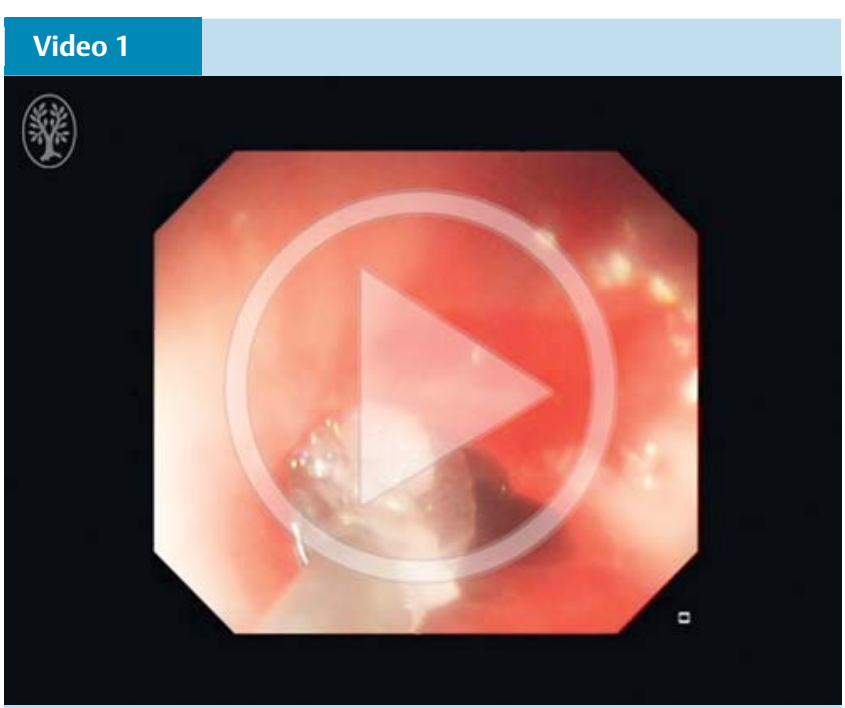

After placement on the device, the epidermal cell sheet (ECS) was transported from the mouth onto the tears after endoscopic balloon dilation (EBD). The release procedure enabled accurate placement of the ECS on to the artificial ulcer after EBD. Online content including video sequences viewable at: http://dx.doi.org/10.1055/s-0042-116145

\section{Macroscopic analysis for competitive performance evaluation}

All animals were observed endoscopically and euthanized at 3 weeks after ESD. Previous animal studies have confirmed the measurement of esophageal stricture in extracted specimens $[8$, 12]. Photographs were taken of each esophageal specimen with a ruler immediately after extraction from pigs. The length of the esophageal specimen was measured by Image software (National Institutes of Health, Bethesda, MD, United States). The rate of esophageal strictures was calculated as $(1-\operatorname{Lmax} / \mathrm{Lnr}) \times 100$, where Lmax and Lnr are the lengths of the short axes at the maximally narrow mucosa and the normal mucosa on the oral side of esophageal specimens, respectively.

\section{Histological analysis}

Esophageal specimens were routinely processed into $3-\mu \mathrm{m}$-thick paraffin-embedded sections. After deparaffinization, the sections were stained with hematoxylin and eosin (HE) and Sirius red, the latter using a Picrosirius Red Stain kit (Polysciences, Inc., Warrington, Pennsylvania, United States). Ulcer sites of muscle atrophy and fibrosis after esophageal strictures were evaluated by Sirius red staining. Fibrosis and atrophy of the muscularis propria (MP) were graded numerically by a modified Honda's scoring system in which 0 was defined as the absence of atrophic or fibrotic changes in any examined sections of the MP, 1 as atrophy 
Table 1 Procedure for transplantation of epidermal cell sheets (ECSs).

\begin{tabular}{|clc|}
\hline Procedure & Transplantation & Procedure time (s) \\
\hline Transplantation 1 & & \\
\hline Procedure 1 & Success & 130 \\
\hline Procedure 2 & Success & 110 \\
\hline Transplantation 2 & & \\
\hline Procedure 3 & Success & 100 \\
\hline Procedure 4 & Success & 125 \\
\hline Procedure 5 & Failure & N/A \\
\hline Procedure 6 & Success & 205 \\
\hline
\end{tabular}

N/A: not available.

or fibrosis present but confined to the partial MP, 2 as atrophy or fibrosis present but confined to the full-thickness MP, and 3 as transmural fibrosis of the MP [13]. Infiltration of inflammatory cells into the ulcer sites of esophageal strictures was evaluated by HE staining. Inflammatory cells were counted in five random high power $(\times 400)$ fields $(\mathrm{HPF})$ per pig.

\section{Engraftment of transplanted epidermal cell sheets}

Before transplantation, harvested ECSs were labeled with PKH26GL (Sigma-Aldrich/Merck, St. Louis, Missouri, United States). A part of the transplanted area of each esophagus was frozen in OCT compound and sectioned at $5 \mu \mathrm{m}$. The sections were stained with 4,6-diamidino-2-phenylindole (DAPI) and observed under a confocal laser microscope (FV1200; Olympus). The closed sections were stained with an anti-pan cytokeratin antibody (MAB9766, Abnova Corporation, Taipei, Taiwan) and DAPI, and monitored by confocal laser microscopy.

\section{Statistical analysis}

Data are expressed as the means \pm standard deviation (SD). The numbers of invading inflammatory cells were compared by the Student's $t$ test. Probability values $(P)$ of less than 0.05 were considered statistically significant. All statistical analyses were performed using the SAS-JMP program for Windows (SAS Institute Inc., Cary, North Carolina, United States).

\section{Results}

$\nabla$

Transplantation of a tissue-engineered ECS after EBD The ECSs produced after culturing for 2 weeks were approximately $20 \mathrm{~mm}$ in diameter ( $\bullet$ Fig. 2c) and had epidermoid features ( $\bullet$ Fig. 2 d).
ESD and EBD were performed safely in all animals ( $\bullet$ Fig. 2 e,f). Six ECS transplantations were performed after EBD in two pigs using the procedures described in Table 1. Of these six ECSs, five were successfully transplanted into tears, two of two in one pig, and three of four in the other, giving a success rate of $83 \%$ for ECS transplantation. The procedure time for ECS transplantation was $134 \pm 41.4 \mathrm{~s}$ (mean $\pm \mathrm{SD}$ ).

\section{Clinical conditions and endoscopic findings}

The clinical conditions and endoscopic findings in the six pigs are summarized in Table 2 and $\bullet$ Fig. 3. Three weeks after ESD, the two control pigs had dysphagia scores of 4 and 3, respectively, which were accompanied by severe strictures and almost closed internal lumens. One of these pigs also showed a marked reduction in body weight. The two pigs that underwent EBD alone showed a decrease in dysphagia a few days after EBD, followed by recurrence. Three weeks after ESD, both pigs in the EBD group had dysphagia scores of 3 . Strictures recurred to the same degree as before EBD, and the internal lumens were narrow. Although the two pigs that underwent ECS transplantation had dysphagia before EBD, the dysphagia was reduced after EBD and ECS transplantation. Three weeks after ESD, the dysphagia scores in these two pigs were 2 and 1, respectively. There were fewer strictures, and internal lumens remained sufficiently wide.

\section{Histological findings}

The macroscopic findings of the three groups are summarized in - Table 1 and Fig. 4. The two control pigs developed severe strictures, with stricture rates of $92.2 \%$ and $87.7 \%$. The pigs that underwent EBD alone were affected by re-strictures with rates of $71.7 \%$ and $78.2 \%$. In contrast, the stricture rates in pigs that underwent ECS transplantation were $55 \%$ and $60 \%$.

Histological evaluation of inflammatory cell infiltration into ulcer sites after ESD showed that the number of inflammatory cells per HPF was significantly lower in pigs that underwent ECS transplantation $(113.2 \pm 53.8$ cells/HPF) than in control pigs (223.6 \pm 73.3 cells/HPF, $P<0.01)$ and in pigs that underwent EBD alone (206.2 \pm 63.9 cells/HPF, $P<0.01$ ).

Fibrosis and atrophy of the MP layer in ulcer sites were evaluated after ESD by Sirius red staining. The MP layer in control pigs showed evidence of transmural fibrosis and atrophy, whereas the MP layers in the other groups did not. Both control pigs had atrophy scores in the MP layer of 3. The two pigs that underwent EBD alone had atrophy scores of 2 and 3, respectively, whereas both pigs that underwent ECS transplantation had atrophy scores of 1 .

Table 2 Assessments of physiological findings and esophageal strictures.

\begin{tabular}{|c|c|c|c|c|c|}
\hline \multirow[t]{2}{*}{ Case } & \multirow[t]{2}{*}{ Weight gain 3 weeks after ESD } & \multicolumn{3}{|c|}{ Dysphagia score } & \multirow[t]{2}{*}{ Rate of strictures, \% } \\
\hline & & Before ESD & 2 weeks after ESD & 3 weeks after ESD & \\
\hline Control 1 & $-1.9 \mathrm{~kg}(-9.9 \%)$ & 0 & 3 & 4 & 92.2 \\
\hline Control 2 & $-0.10 \mathrm{~kg}(-0.6 \%)$ & 0 & 3 & 3 & 87.7 \\
\hline EBD 1 & $+0.0 \mathrm{~kg}(+0.0 \%)$ & 0 & 3 & 3 & 71.7 \\
\hline EBD 2 & $-0.7 \mathrm{~kg}(-3.5 \%)$ & 0 & 4 & 3 & 78.2 \\
\hline Transplantation 1 & $+0.0 \mathrm{~kg}(0.0 \%)$ & 0 & 3 & 2 & 55.0 \\
\hline Transplantation 2 & $+1.1 \mathrm{~kg}(+6.2 \%)$ & 0 & 4 & 1 & 60.0 \\
\hline
\end{tabular}

ESD, endoscopic submucosal dissection; EBD, endoscopic balloon dilation. 


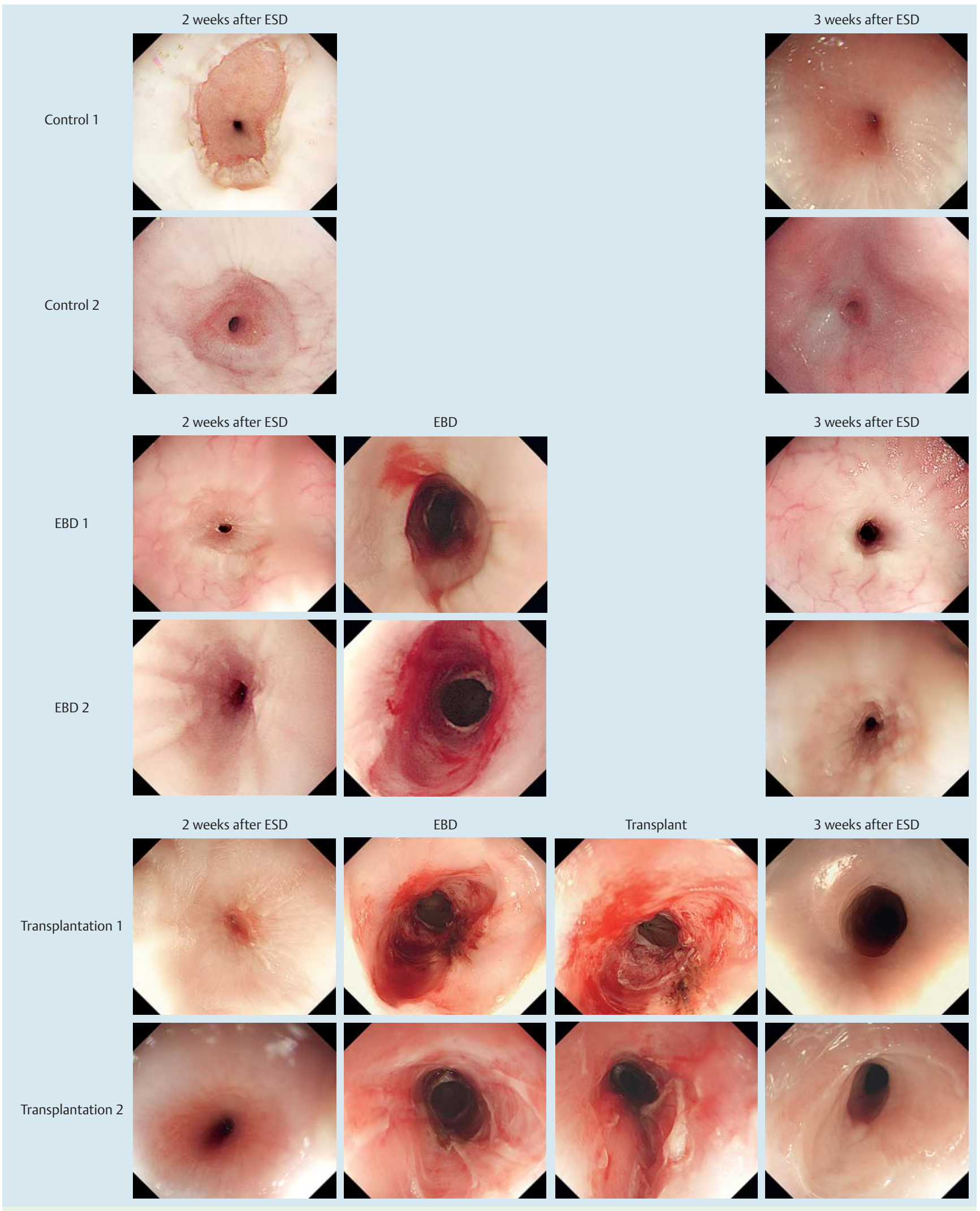

Fig. 3 Chronological endoscopic findings at 2 weeks and 3 weeks after esophageal endoscopic submucosal dissection (ESD). The two control pigs developed severe esophageal strictures at 3 weeks after ESD. The two pigs that underwent endoscopic balloon dilatation (EBD) alone developed re-strictures at 1 week after EBD, whereas the two pigs that underwent epidermal cell sheet (ECS) transplantation after EBD did not. 


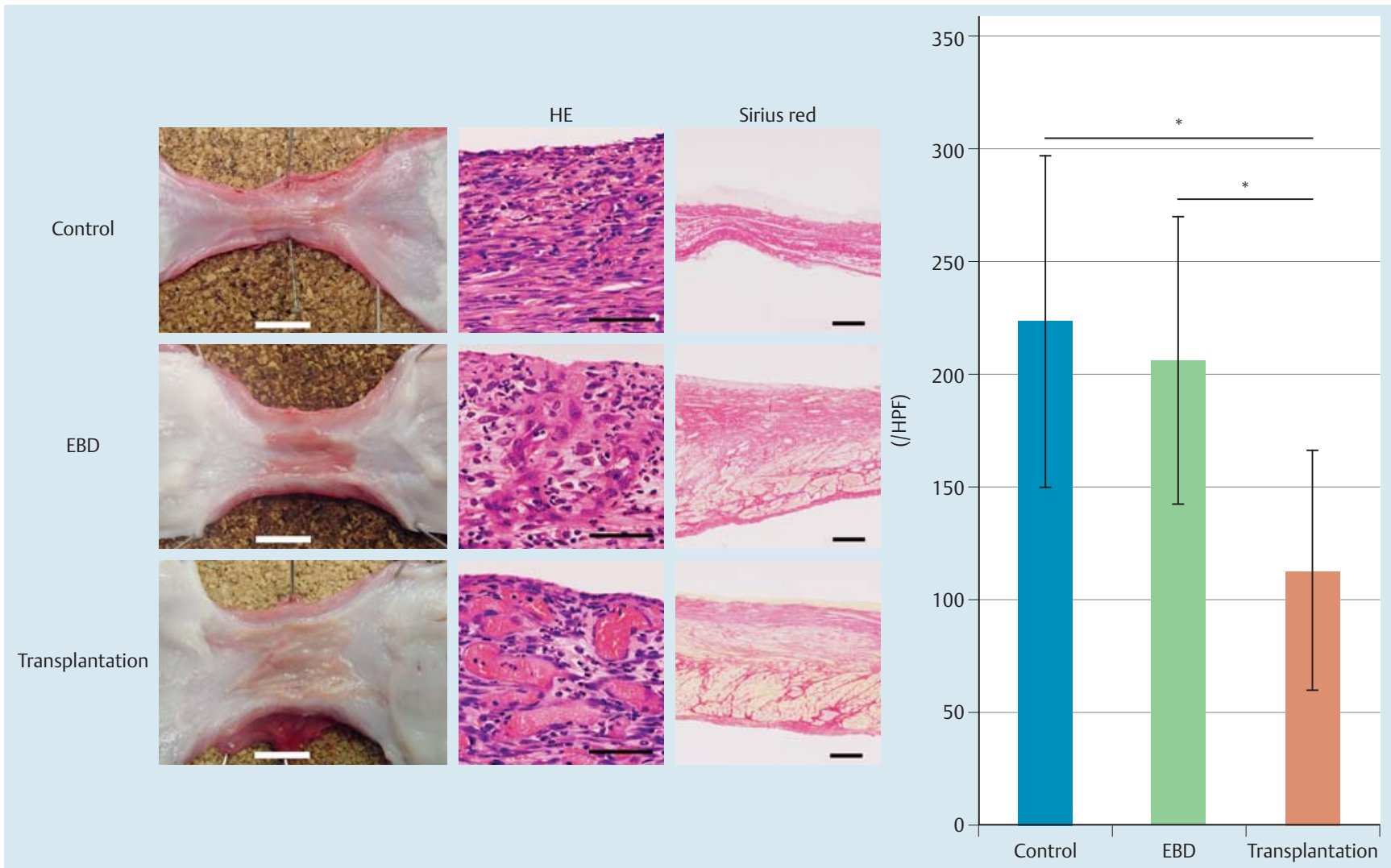

Fig. 4 Histological findings. Macroscopic findings revealed severe strictures in control pigs, re-strictures in pigs that underwent endoscopic balloon dilation (EBD) alone, and the absence of re-strictures in pigs that underwent EBD followed by epidermal cell sheet (ECS) transplantation. White bars indicate $10 \mathrm{~mm}$. H\&E staining showed infiltration of inflammatory cells in all pigs (black bars indicate $50 \mu \mathrm{m}$ ). Sirius red staining revealed muscle atrophy and fibrosis in all pigs (black bars indicate $500 \mu \mathrm{m}$ ). The graph shows the numbers of inflammatory cells per high power field (HPF). ${ }^{*} P<0.01$.

\section{Engraftment of transplanted ECSs}

PKH-labeled cells were observed in the ulcer sites after transplantation of ECSs. In addition, keratinocytes, which are positive for pan-cytokeratin, were observed in the ulcer sites after ECS transplantation ( $\bullet$ Fig. 5).

\section{Discussion}

Using our developed procedure, ECSs were transplanted into esophageal tears resulting from EBD. This transplantation method was successful while avoiding re-strictures. Taken together, these results indicate that the combination of EBD and the transplantation of bioengineered ECSs may be a promising new approach to prevent the development of refractory esophageal strictures after ESD.

This study involved the artificial creation of long esophageal strictures $(>20 \mathrm{~mm})$ in a porcine model. A small clinical study found that strictures of this length are at high risk of becoming refractory [14]. Therefore, this animal model is similar to refractory esophageal strictures observed in human patients. Moreover, the EBD procedure used in this study was developed for an animal model. To avoid perforation and deep ulceration, the locations and depths of the tears were equalized relative to the four slits of the mucosa on post-ESD esophageal strictures before EBD. Methods to prevent or treat severe esophageal strictures after endoscopic removal of large tumors remain unclear. Risk factors for esophageal strictures after ESD include a cervical location, a tumor size greater than $3 / 4$ of the esophageal circumference, and a longitudinal tumor diameter of $>40 \mathrm{~mm}$ [15-17]. Almost all patients who undergo complete circumferential esophageal ESD develop post-ESD strictures. Although several treatments have been developed to prevent refractory esophageal strictures, it may be optimal to select the least invasive stepwise treatments [3]. Generally, EBD is the first choice for the management of esophageal strictures [5]. Local injection of triamcinolone acetonide and systemic steroid treatment have been shown to be effective to reduce the number of EBD sessions after stricture development, thus preventing esophageal strictures from becoming refractory [18-20]. However, this treatment method has several disadvantages including a delay in tissue remodeling and depression of the immune system [18 - 20]. Local injection of mitomycin $C$ after EBD has also been reported to be effective and safe in the prevention of refractory esophageal strictures [21].

After development of refractory esophageal strictures, more invasive treatments are required. Temporary metallic stents are effective for long periods of time, although they may have adverse effects, including pain, nausea, and deviation, requiring stent removal $[4,22]$. Hyperplastic reactions to inserted metallic stents, although rare, can cause fistulas. Biodegradable stents may reduce the disadvantages of metallic stents, although foreign-body reactions and stent migration may still occur [6]. Radical incision and cutting methods (RIC), resulting in endoscopic volume reduction of extensive granulation, has been reported to reduce treatment periods [23]. Because they are highly invasive, surgical procedures, including esophageal resection and reconstruction, 

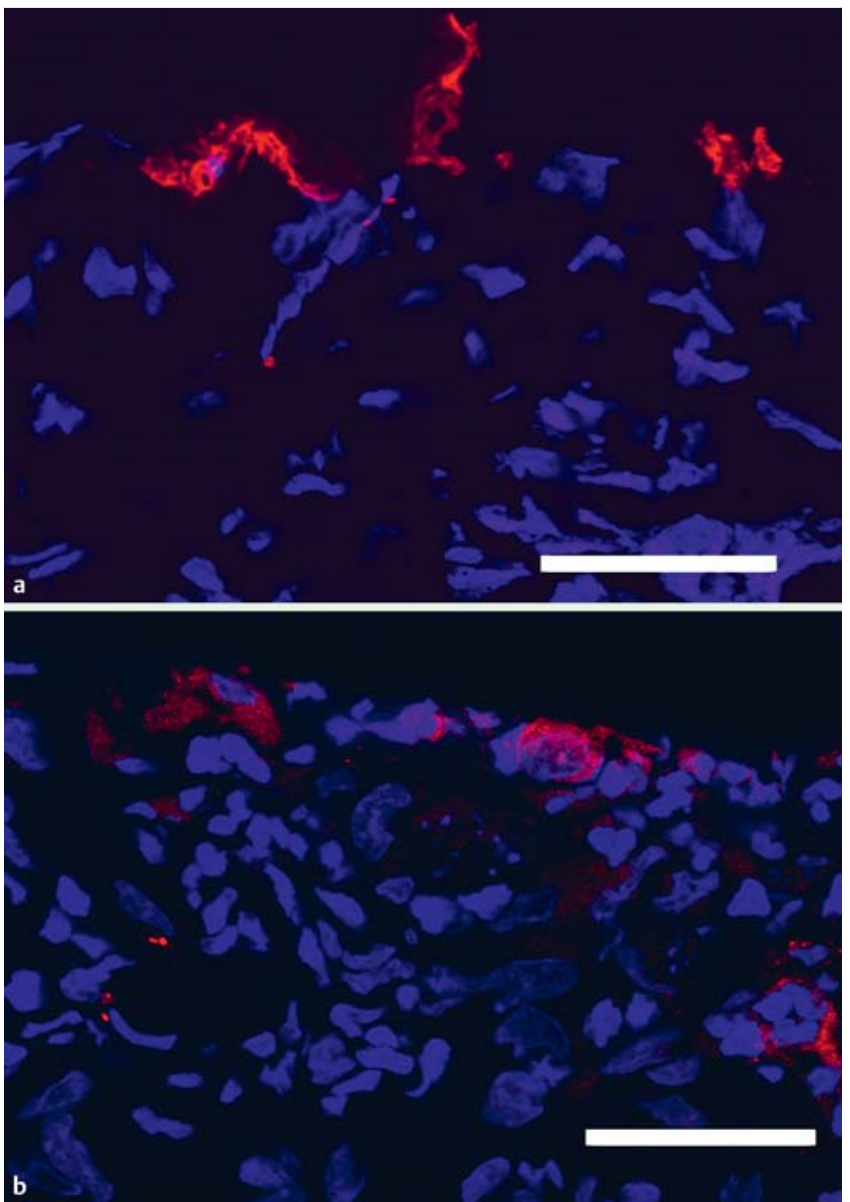

Fig. 5 Engraftment of transplanted epidermal cell sheets (ECSs). a PKHlabeled cells were observed in ulcer sites after endoscopic balloon dilation (EBD). Scale bar indicates $50 \mu \mathrm{m}$ (red, PKH; blue, DAPI). b Colonies of epithelial cells (red) were observed in ulcer sites after EBD. Scale bar indicates $50 \mu \mathrm{m}$ (red, pan-CK; blue, DAPI).

are the final approach for treating refractory esophageal strictures. Dilatation surgery using a scaffold patch was recently reported to be safe and effective in small numbers of patients [24]. Despite these procedures being invasive and having limited efficacy, they are required to overcome developed refractory esophageal strictures [25].

Transplantation of ECSs immediately after ESD was previously shown to be effective in the prevention of post-ESD strictures $[7,8]$. The results presented here also indicate that ECS transplantation after EBD can prevent re-strictures. However, the conventional method of ECS transplantation using a support membrane is technically difficult, particularly in narrow sections, because the support membrane consists of a solid material, and ECSs tend to become wedged and do not fit well in these tears. Our novel ECS transplantation procedure to solve these issues resulted in the transplanted ECSs easily attaching by their basal surfaces to ulcer sites within a few minutes after EBD [26]. Engraftment of these ECSs at ulcer sites prevented re-adhesion of the wound bed after EBD.

Interestingly, these transplanted ECSs also inhibited the inflammation of ulcer sites and prevented the atrophy and fibrosis of MP layers. The number of inflammatory cells infiltrating into esophageal ulcer sites was much lower in pigs that underwent transplantation than in control and EBD-treated pigs. Moreover, control pigs and those that underwent EBD alone showed trans- mural muscle atrophy and fibrosis after esophageal ESD. These results demonstrate the importance of protection of the muscle layer from inflammation at ulcer sites. The efficacy of ECS transplantation with EBD may be limited because esophageal motility and muscle compliance may not be preserved in chronic strictures such as those caused by caustic agents and radiotherapy. Thus, the efficacy of ECS transplantation with EBD may be maximized to preserve the muscle layer to prevent re-strictures under conditions in which refractory strictures or chronic strictures develop.

This study had several limitations. First, it was designed as a preliminary animal study to test the efficacy and safety of the ECS transplantation procedure to prevent re-strictures after EBD. Previous studies, which have already shown efficacy to prevent strictures after esophageal ESD, indicate that therapeutic effects of ECS transplantation after EBD can be expected, on the condition that ECS transplantation succeeds [7-9].

ECS transplantation procedures must be clinically evaluated in humans to confirm and validate these promising preliminary results. Clinical comparisons of EBD with and without ECS transplantation are also needed.

In conclusion, this pilot study provides information to plan future studies on the efficacy of ECS transplantation with EBD to prevent esophageal re-strictures. Preliminary results indicate the stability of the ECS transplantation procedure and the engraftment of transplanted ECS in tears after EBD.

Competing interests: Masayuki Yamato is a consultant and stockholder of CellSeed. None of the other authors have any financial relationships relevant to this publication to disclose.

\section{Institutions}

'Department of Surgery, Graduate School of Biomedical Sciences, Nagasaki University, Nagasaki, Japan

2 Institute of Advanced Biomedical Engineering and Science, Tokyo Women's Medical University, Tokyo, Japan

${ }^{3}$ Laboratory for Integrated Biodevice, Quantitative Biology Center - RIKEN, Fukita, Japan

${ }^{4}$ Department of Pharmaceutical Sciences, Graduate School of Pharmaceutical Science, Tokyo University of Science, Noda, Japan

\section{Acknowledgments}

The authors thank Norio Iwata, Terumasa Umemoto, and Teruo Okano for their constructive suggestions. The authors also thank Peter Elbe and Eduard Jonas, who are endoscopists in Kalorinska University Hospital, for constructive suggestions on devices. Hiroyuki Morimoto and Tomonori Yano have great experience and knowledge of esophageal strictures and provided clinical questions. The authors thank Ichirei Takeda, who cared for the experimental animals and developed the anesthesia care protocols. This study was partially supported by a Grant-in-Aid for Scientific Research (14483609 Nobuo Kanai, 15H06503 Shinichiro Kobayashi); by the Creation of Innovation Centers for Advanced Interdisciplinary Research Areas Program in the Project for Developing Innovation Systems "Cell Sheet Tissue Engineering Center (CSTEC)" from the Ministry of Education, Culture, Sports, Science and Technology (MEXT). 


\section{References}

1 Kobayashi S, Kanai N, Ohki T et al. Prevention of esophageal strictures after endoscopic submucosal dissection. World J Gastroenterol 2014; 20: $15098-15109$

2 Sun F, Yuan P, Chen T et al. Efficacy and complication of endoscopic submucosal dissection for superficial esophageal carcinoma: a systematic review and meta-analysis. J Cardiothorac Surg 2014; 9: 78

3 de Wijkerslooth LR, Vleggaar FP, Siersema PD. Endoscopic management of difficult or recurrent esophageal strictures. Am J Gastroenterol 2011; 106: 2080 - 2091

4 Hirdes MM, Siersema PD, Vleggaar FP. A new fully covered metal stent for the treatment of benign and malignant dysphagia: a prospective follow-up study. Gastrointest Endosc 2012; 75: 712 - 718

5 Yano T, Yoda Y, Satake $H$ et al. Radial incision and cutting method for refractory stricture after nonsurgical treatment of esophageal cancer. Endoscopy 2013; 45: 316-319

6 Ham YH, Kim GH. Plastic and biodegradable stents for complex and refractory benign esophageal strictures. Clin Endosc 2014; 47: 295 - 300

7 Ohki T, Yamato M, Murakami D et al. Treatment of oesophageal ulcerations using endoscopic transplantation of tissue-engineered autologous oral mucosal epithelial cell sheets in a canine model. Gut 2006; 55: $1704-1710$

8 Kanai $N$, Yamato M, Ohki $T$ et al. Fabricated autologous epidermal cell sheets for the prevention of esophageal stricture after circumferential ESD in a porcine model. Gastrointest Endosc 2012; 76: 873-881

9 Ohki T, Yamato M, Ota $M$ et al. Prevention of esophageal stricture after endoscopic submucosal dissection using tissue-engineered cell sheets. Gastroenterology 2012; 143: 582-588

10 Takagi R, Murakami D, Kondo $M$ et al. Fabrication of human oral mucosal epithelial cell sheets for treatment of esophageal ulceration by endoscopic submucosal dissection. Gastrointest Endosc 2010; 72: $1253-1259$

11 Mellow MH, Pinkas H. Endoscopic laser therapy for malignancies affecting the esophagus and gastroesophageal junction. Analysis of technical and functional efficacy. Arch Intern Med 1985; 145: 1443-1446

12 Honda $M$, Hori Y, Nakada $A$ et al. Use of adipose tissue-derived stromal cells for prevention of esophageal stricture after circumferential EMR in a canine model. Gastrointest Endosc 2011; 73: 777-784

13 Honda M, Nakamura T, Hori Y et al. Process of healing of mucosal defects in the esophagus after endoscopic mucosal resection: histological evaluation in a dog model. Endoscopy 2010; 42: $1092-1095$
14 Yoda Y, Yano T, Kaneko K et al. Endoscopic balloon dilatation for benign fibrotic strictures after curative nonsurgical treatment for esophageal cancer. Surg Endosc 2012; 26: 2877-2883

15 Ono S, Fujishiro M, Niimi K et al. Long-term outcomes of endoscopic submucosal dissection for superficial esophageal squamous cell neoplasms. Gastrointest Endosc 2009; 70: 860-866

16 Mizuta H, Nishimori I, Kuratani Y et al. Predictive factors for esophageal stenosis after endoscopic submucosal dissection for superficial esophageal cancer. Dis Esophagus 2009; 22: 626-631

17 Lewis JJ, Rubenstein JH, Singal AG et al. Factors associated with esophageal stricture formation after endoscopic mucosal resection for neoplastic Barrett's esophagus. Gastrointest Endosc 2011; 74: 753 - 760

18 Kochhar R, Ray JD, Sriram PV et al. Intralesional steroids augment the effects of endoscopic dilation in corrosive esophageal strictures. Gastrointest Endosc 1999; 49: 509-513

19 Yamaguchi $N$, Isomoto H, Nakayama T et al. Usefulness of oral prednisolone in the treatment of esophageal stricture after endoscopic submucosal dissection for superficial esophageal squamous cell carcinoma. Gastrointest Endosc 2011; 73: 1115-1121

20 Hashimoto S, Kobayashi M, Takeuchi M et al. The efficacy of endoscopic triamcinolone injection for the prevention of esophageal stricture after endoscopic submucosal dissection. Gastrointest Endosc 2011; 74: $1389-1393$

21 Machida $H$, Tominaga K, Minamino $H$ et al. Locoregional mitomycin $C$ injection for esophageal stricture after endoscopic submucosal dissection. Endoscopy 2012; 44: 622-625

22 Talreja JP, Eloubeidi MA, Sauer BG et al. Fully covered removable nitinol self-expandable metal stents (SEMS) in malignant strictures of the esophagus: a multicenter analysis. Surg Endosc 2012; 26: 1664-1669

23 Muto M, Ezoe Y, Yano T et al. Usefulness of endoscopic radial incision and cutting method for refractory esophagogastric anastomotic stricture (with video). Gastrointest Endosc 2012; 75: 965 - 972

24 Nieponice A, Ciotola FF, Nachman F et al. Patch esophagoplasty: esophageal reconstruction using biologic scaffolds. Ann Thorac Surg 2014 97: $283-288$

25 Yu JP, Liu YJ, Tao YL et al. Prevention of esophageal stricture after endoscopic submucosal dissection: a systematic review. World J Surg 2015; 39: $2955-2964$

26 Yamato $M$, Utsumi $M$, Kushida $A$ et al. Thermo-responsive culture dishes allow the intact harvest of multilayered keratinocyte sheets without dispase by reducing temperature. Tissue Eng 2001; 7: 473 480 